\title{
Alterations in Team Physical Performance and Possession in Elite Gaelic Football Competition
}

\author{
Declan Gamble ${ }^{1,2, ~ *, ~ N i a l l ~ M o y n a ~}{ }^{2}$, Richard McCann ${ }^{1}$, Damian Martin ${ }^{1}$, Gerard McMahon $^{3}$, \\ Lee Rooney ${ }^{3}$, Matt Spencer ${ }^{4}$, Johnny Bradley ${ }^{5}$, Andrew McCarren ${ }^{6}$ \\ ${ }^{1}$ Sport Northern Ireland Sports Institute, Ulster University, Newtownabbey, Ireland \\ ${ }^{2}$ School of Health and Human Performance, Dublin City University, Dublin, Ireland \\ ${ }^{3}$ School of Sport, Ulster University, Newtownabbey, Ireland \\ ${ }^{4}$ Department of Sport Science and Physical Education, University of Agder, Kristiansand, Norway \\ ${ }^{5}$ Centre of Performance Analysis, Institute of Technology Carlow, Carlow, Ireland \\ ${ }^{6}$ Insight Centre for Data Analytics, Dublin City University, Dublin, Ireland
}

Email address:

declangamble@sportni.net(D. Gamble)

${ }^{*}$ Corresponding author

To cite the article:

Declan Gamble, Niall Moyna, Richard McCann, Damian Martin, Gerard McMahon, Lee Rooney, Matt Spencer, Johnny Bradley, Andrew McCarren. Alterations in Team Physical Performance and Possession in Elite Gaelic Football Competition. American Journal of Sports Science. Vol. 8, No. 2, 2020, pp. 39-48. doi: 10.11648/j.ajss.20200802.13

Received: June 2, 2020; Accepted: June 18, 2020; Published: July 13, 2020

\begin{abstract}
Differences in performance between winning and losing were examined in 1 elite Gaelic football team in 20 games across 2 complete competitive seasons. Possession was codified using Dartfish TeamPro software and distance covered; walking, jogging, running, and running at high and maximum speeds, was evaluated using Catapult OptimEye S5 player tracking devices. Distance covered in low intensity activity (LIA, $\left.<4.0 \mathrm{~m} \cdot \mathrm{s}^{-1}\right)$, high intensity running $\left(\mathrm{HIR}, \geq 4.0 \mathrm{~m} \cdot \mathrm{s}^{-1}\right)$ and very high intensity running (VHIR, $\geq 5.5 \mathrm{~m} \cdot \mathrm{s}^{-1}$ ) was also examined along with PlayerLoad ${ }^{\mathrm{TM}}$, which represented a composite of all accelerations. Data from 53 players ( $\mathrm{n}=405$ files) was collated into specific match periods to facilitate a temporal analysis between the first and second halves and from quarter $1(\mathrm{Q} 1)$ to quarter $4(\mathrm{Q} 4)$, with significance accepted at $p \leq 0.05$. Total distance and running was higher in games lost, whereas total distance, walking and LIA was higher in halves lost. Only walking was higher in quarters lost. The percentage of possession declined in halves and quarters lost. In games lost, high speed running declined in the second half. From Q1 to Q4; PlayerLoad ${ }^{\mathrm{TM}}$, total distance, jogging, high speed running, HIR and VHIR, decreased in all games combined and in games lost. Possession frequency declined in Q4 in all games and in games won. Overall, total distance was higher in games lost and physical performance declines were more pronounced when examined by match quarter compared to half and were only apparent in games lost. Similarly, reductions in possession frequency and percentage were more evident when examined by quarter or period lost, respectively. These findings can inform the prescription of conditioning and field-training strategies to mitigate the reductions in performance observed in losing and towards the end of games.
\end{abstract}

Keywords: Performance Analysis, Technical, Tactical, GPS, Temporal

\section{Introduction}

Gaelic football is an invasive field-based team sport played in Ireland. Although the sport retains its amateur status, the players adopt a quasi-professional training schedule [1]. Field practice and gym conditioning are conducted by teams in preparation for inter-county competition, which formally begins in January with the
National Football League (NFL) and concludes in August following the completion of the All- Ireland Championship (AIC). Match-play is characterised by turnovers and fast paced-transitions, as teams gain possession, attack or counterattack and try to score in the opposition's defensive zone [2]. Consequently, significant physical capacities are required to sustain intermittent high intensity activities incorporating; accelerations, decelerations and changes in 
direction, and high- and maximal-speed running, although these are often interspersed with low intensity recovery periods. Physical contact occurs throughout games, aggravated by man-to-man marking [3], shoulder charging and tackling [4], and particular intense contests can ignite the passion of players and spectators alike. However, these impacts may exacerbate high levels of fatigue [5] and contribute to declines in physical performance [4], potentially affecting match outcome. Therefore, to understand specific aspects of performance contributing to match outcome [6], it is appropriate to analyse a combination of; physical, tactical and technical indicators demonstrated by successful (winning) and unsuccessful (losing) teams.

The player tracking technology incorporating GPS, used extensively in team sports to provide comprehensive analysis of physical performance during training and competition [7], is now embedded within the preparation programmes and performance analysis of most elite Gaelic football teams. This has facilitated examination of the activity and running profiles of players, with recent studies demonstrating average total distances covered, ranging from $\sim 8.2$ to $8.9 \mathrm{~km}$ [8-10], and mean peak speeds, ranging from $\sim 7.8$ to $8.4 \mathrm{~m} \cdot \mathrm{s}^{-1}[4,9]$. Moreover, in a study exploring the relationship between running performance and technical variables, persistent fouling in the middle third was recently shown to have the largest negative impact on running, whereas the percentage of short kick outs performed by the opposition and total opposition possession time positively increased the total distance and high speed distance ran [11]. Hierarchical differences and temporal decrements in positional running [8, 9], and activity profiles, PlayerLoad ${ }^{\mathrm{TM}}$ and heart rate responses [4] have also been identified. Additionally, players from higher ranked teams covered more distance at high speed $\left(>\sim 4.7 \mathrm{~m} \cdot \mathrm{s}^{-1}\right)$ compared to lower ranked players [10, 12], perhaps due to superior levels of conditioning. In Q4, players ran significantly less high-speed distance in big losses, defined as $>6$ points, compared to draws and wins [12], which highlights the importance of considering situational [13] and motivational factors [14] when interpreting performance data. Also, players competing in the latter stages of the AIC (i.e. August and September) covered significantly more total distance and distance at high speed in Q4 compared to all other months [15], suggesting that progressive conditioning and the enhanced profile of the AIC competition may facilitate superior levels of physical performance compared to those observed earlier in the season. Unfortunately, in these studies interpretation of the physical data may be limited due to the absence of information relating to temporal changes in technical performance.

Recent investigations in Gaelic football identified team tactical and technical performance indicators that discriminated between winning and losing, through analysis of data from full-games [2, 16] or halves and quarters [17, 18]. The importance of possession was highlighted as winners were more effective at regaining and retaining possession, and converted more scores per 10 possessions compared to losers [2]. Moreover, possession was found to contribute significantly more to winning in the second compared to the first half [18]. Although, a significant decline in possession was observed in both winners and losers, when comparing Q1 to Q4 [17]. Unfortunately, there were no physical analyses conducted to contextualise the technical results, therefore it is unclear whether this decline in possession was due to physical fatigue and/or contextual factors [19].

The physical and technical performance profile obtained from a team during match play is likely influenced by; their player's prior experience, training age and fitness level, the context (home or away), competition status and importance (league or championship) of the game, stage of season (early, mid, late), level of opposition, and tactical strategy employed $[10,19,20]$. Many of these factors are considered in the development of the tactical plans communicated by the coach, rehearsed in training and implemented during games. These strategies vary in the formations employed and roles in which players are deployed [2, 17], with most contemporary Gaelic football coaches having discarded the traditional rigid configuration of 6 defenders, 2 midfielders and 6 attacking players, and adopted tactics that facilitate a more dynamic approach to defensive and offensive play. Consequently, the starting location of the player(s) on the pitch may not reflect the tactical role and associated physical performance required. Moreover, execution of a game plan requires players to possess high levels of physical conditioning to maintain sufficient performance levels. Importantly, if performance levels decline, coaches can also use their substitution options to positively impact the dynamic or momentum of play, by introducing new players to enhance the organisation, physical profile, and/or creativity of their teams.

Despite the recent studies documenting the technical performance of teams and activity profiles of Gaelic football players, there is currently no published information pertaining to the physical performance indices of elite teams. As match derived performance data is analysed and used to inform the physical preparation of players through prescription of specific training, obtaining running profiles and possession characteristics is key to interpreting match data appropriately. Therefore, the aim of this study was to examine differences in the physical performance and possession characteristics of a Gaelic football team in winning compared to losing. A secondary aim was to evaluate temporal changes in physical performance and possession across match halves and from Q1 to Q4 in relation to winning and losing.

\section{Methods}

\subsection{Participants}

In this observational study, physical performance indicators and possession characteristics from 1 elite 
Gaelic football team were examined in 22 games throughout 2 competitive seasons. This (reference) team competed against 13 opposition teams during 16 intercounty Division 1 NFL and 6 AIC games (win $=8$, loss $=$ 12 , draw $=2$ ). As winners and losers could not be differentiated from matches which ended in a draw, 2 games were excluded and the final analysis involved 20 games (reference team vs. 12 teams). A small winning or losing margin of $\leq 5$ points was associated with 11 games, whereas the remaining 9 games involved a large win/loss (between 6-15 points). Data from 51 outfield players and 2 goalkeepers (mean $\pm \mathrm{SD}$; age, $24.5 \pm 3.6 \mathrm{y}$; height, 181.9 $\pm 5.3 \mathrm{~cm}$; mass, $83.5 \pm 7.2 \mathrm{~kg}$; estimated $\dot{\mathrm{V} O} 2 \max , 56.5 \pm$ $3.3 \mathrm{ml} \cdot \mathrm{kg}^{-1} \cdot \mathrm{min}^{-1}$ ) were examined, incorporating 405 individual game files. The experimental procedures were approved by the University Research Ethics Committee. Participants were provided with a plain language statement outlining the nature and demands of the study as well as the inherent risks. Written informed consent was obtained prior to participation.

\subsection{Procedures}

The experimental procedures used in this investigation have been documented previously [2, 4]. Microtechnology devices (Optim Eye S5, Catapult Sports, Australia) containing GPS $(10 \mathrm{~Hz})$ and triaxial accelerometers $(100 \mathrm{~Hz})$ were used to investigate the activity profiles and PlayerLoad ${ }^{\mathrm{TM}}$ of the players. Locomotor activities $\left(\mathrm{m} \cdot \mathrm{s}^{-1}\right)$ were collated and classified as; standing $(\geq 0.00-<0.19)$, walking $(\geq 0.19-<2.00)$, jogging $(\geq 2.00-<4.00)$, running $(\geq 4.00-<5.50)$, high speed running $(\geq 5.50-<7.00)$, and maximal speed running $(\geq 7.00)$, resembling activity profiles and thresholds reported previously in Australian football [19, 21, 22], hurling [23], and soccer [24, 25]. Other match measures included; low intensity activity (LIA, i.e. standing, walking and jogging, $<4.0 \mathrm{~m} \cdot \mathrm{s}^{-1}$ ), high intensity running (HIR, $\geq 4.0 \mathrm{~m} \cdot \mathrm{s}^{-1}$ ), very-high intensity running (VHIR, $\geq 5.5$ $\mathrm{m} \cdot \mathrm{s}^{-1}$ ) and PlayerLoad ${ }^{\mathrm{TM}}$. This index of external load was calculated as the square root of the sum of the squared instantaneous rate of change in acceleration in the forward, vertical and sideward directions and divided by a scaling factor of 100 [26].

Data from each game was downloaded using the Catapult Sprint (v5.1.7) software, exported into Microsoft Excel and transformed for evaluation. The mean $( \pm \mathrm{SD})$ number of GPS satellites acquired during the first and second halves was 13.8 \pm 1.4 and $13.8 \pm 1.1$, respectively. The corresponding mean $( \pm \mathrm{SD}$ ) horizontal dilution of precision scores of $0.67 \pm 0.15$ and $0.68 \pm 0.17$ for the first and second halves, reflected the geometrical arrangement of the satellites and indicated the acceptable accuracy of the signal [27]. The data from all starting and substitute players were collated for the full duration of their involvement in each game to enable team performance to be evaluated. A small number of data files $(n=11,0.03 \%)$ were unusable due to; a player removing the tracking device following the warm-up or during play $(n=3)$, the device stopped working $(n=5)$, or the file was corrupted and unreadable $(n=3)$. Data was estimated for 1 player who did not wear the device during the game by using the results from another player from the same positional line. Results for the remaining 10 players were estimated using their own relative data from useable match periods. Overall, $\sim 1.53 \%$ of the GPS and $\sim 0.78 \%$ of the accelerometer data was estimated. The reliability and validity of the player tracking technology used in this study to quantify velocity, distance and PlayerLoad ${ }^{\mathrm{TM}}$ has been reported previously [28, 29]. In addition, internal observations utilising a protocol similar to that outlined previously [30] were used to validate the OptimEye S5 player tracking devices. The bias for estimating total distance in each trial $(\mathrm{n}=86)$ of a $135 \mathrm{~m}$ team sports specific circuit was trivial at $1.5+0.3 \%$ versus the criterion method (trundle wheel).

In relation to technical evaluation, match footage from internal team video recordings and from external media broadcasters was imported and coded using a custom built tagging panel in Dartfish (v8) TeamPro software (Fribourg, Switzerland). Following data validation, the coding events were then exported into Microsoft Excel (Microsoft, USA) and transformed to facilitate analysis. To determine intrarater reliability for possession characteristics, two separate games were randomly selected and coded twice over a 4week period. Using a convention described previously [31], a two-way mixed effects model, evaluating absolute agreement between the mean of; 4 full games, 8 halves or 16 quarter measurements, was selected to compute the intraclass correlation coefficient (ICC). A mean ICC of 1.00 was recorded for the frequency and percentage of team possessions across full games, halves and quarters, demonstrating excellent reliability [32].

\subsection{Analysis}

Data analyses were performed using the Statistical Package for Social Science (SPSS) software version 24 (IBM SPSS Inc, Chicago, IL, USA). Normality of distribution for all variables was assessed using the Shapiro-Wilk test. Differences in team performance indicators were evaluated throughout full games, halves and quarters, using an independent t-test to compare results from winning with losing. In addition, relative differences between the first and second halves and between Q1 and Q4 were analysed irrespective of match outcome and then in relation to winning and losing using a one-sample t-test. Descriptive statistics are presented as mean $\pm \mathrm{SD}$ and a $p$ value of $\leq 0.05$ was considered statistically significant.

\section{Results}

\subsection{Match Characteristics}

The average playing time (mean $\pm \mathrm{SD}$ ) for the full games $(n=20)$, halves $(n=38)$ and quarters $(n=68)$ was 73:59 $\pm 1: 39$, 37:01 $\pm 1: 04$, and 18:28 $\pm 0: 31 \mathrm{~min}: \mathrm{s}$, respectively. The related ball in play times were 37:05 $\pm 3: 41,18: 25 \pm 2: 02$, and 9:12 $\pm 1: 13$ min:s, respectively. In all games combined, there was no significant differences in playing time between 
the first and second halves (36:49 \pm 0:57 vs. 37:10 \pm 1:09 min:s). The time of ball in play was significantly higher ( $p=$ $0.023)$ in the second half $(18: 03 \pm 1: 53$ vs. 19:02 $\pm 2: 12$ min:s). There was no significant difference between Q1 and Q4 in either playing time (18:25 $\pm 0: 28$ vs. 18:35 $\pm 0: 34$ min:s) or ball in play time $(9: 25 \pm 1: 13$ vs. $8: 51 \pm 1: 14$ min:s). There were no significant differences in either total playing time or ball in play times between match periods in the 8 games won. In the 12 games lost, there was a significant increase in playing time $(36: 38 \pm 0: 50$ vs. $37: 27 \pm$ $1: 06$ min:s, $p=0.028)$ and decrease in ball in play time $(19: 35 \pm 2: 17$ vs. $18: 06 \pm 1: 58 \mathrm{~min}: \mathrm{s}, p=0.004)$ between the first and second halves. Playing time was significantly higher during Q4 than Q1 (18:20 \pm 0:24 vs. 18:43 \pm 0:33 min:s, $p=$ 0.027 ) in the 12 games lost, although there was a non- significant decrease in ball in play time $(9: 40 \pm 1: 09$ vs. 8:55 $\pm 1: 19$ min:s, $p=0.085$ ).

\subsection{Match Period Summary}

The team performed significantly more running ( $p=$ $0.022)$ and covered a significantly greater total distance $(p=$ 0.028 ) in losing compared to winning full games (Table 1). A significantly greater total distance was covered when losing halves $(p=0.025)$ and this was associated with a significant increase in overall LIA ( $p=0.021)$ and walking ( $p=0.018)$. Walking distance was also significantly higher in losing quarters $(p=0.044)$. There was a significant decline in team possession $(\%)$ in losing halves $(p=0.003)$ and quarters $(p=$ 0.003).

Table 1. Team physical performance and possession in relation to match outcome, across all periods, mean $\pm S D$.

\begin{tabular}{|c|c|c|c|}
\hline Period & Full Games & & \\
\hline Variable & All $(n=20)$ & Win $(n=8)$ & Lose $(n=12)$ \\
\hline PlayerLoad ${ }^{\mathrm{TM}}(\mathrm{AU})$ & $10545 \pm 547$ & $10310 \pm 408$ & $10701 \pm 587$ \\
\hline Stand $(\mathrm{m})$ & $604 \pm 157$ & $643 \pm 224$ & $577 \pm 92$ \\
\hline Walk (m) & $39036 \pm 5517$ & $36850 \pm 5866$ & $40493 \pm 4989$ \\
\hline $\operatorname{Jog}(\mathrm{m})$ & $38592 \pm 2168$ & $37728 \pm 2028$ & $39168 \pm 2144$ \\
\hline Run (m) & $20919 \pm 1575$ & $19964 \pm 1278$ & $21556 \pm 1464^{\alpha}$ \\
\hline High speed run (m) & $9469 \pm 1268$ & $9061 \pm 924$ & $9742 \pm 1425$ \\
\hline Maximum speed run (m) & $2224 \pm 575$ & $2322 \pm 491$ & $2158 \pm 637$ \\
\hline Low intensity activity (m) & $78231 \pm 6132$ & $75221 \pm 5952$ & $80238 \pm 5605$ \\
\hline High intensity running (m) & $32612 \pm 2939$ & $31346 \pm 2011$ & $33456 \pm 3226$ \\
\hline Very-high intensity running (m) & $11693 \pm 1684$ & $11383 \pm 1118$ & $11900 \pm 1997$ \\
\hline Team possession $(\%)$ & $52.8 \pm 3.4$ & $54.6 \pm 3.5$ & $51.6 \pm 3.0$ \\
\hline
\end{tabular}

Table 1. Continue.

\begin{tabular}{|c|c|c|c|c|c|c|}
\hline Period & Halves & & & Quarters & & \\
\hline Variable & All $(n=38)$ & Win $(n=19)$ & Lose $(n=19)$ & All $(n=68)$ & Win $(n=34)$ & Lose $(n=34)$ \\
\hline PlayerLoad ${ }^{\mathrm{TM}}(\mathrm{AU})$ & $5251 \pm 304$ & $5202 \pm 310$ & $5300 \pm 297$ & $2631 \pm 187$ & $2629 \pm 180$ & $2633 \pm 197$ \\
\hline Stand $(m)$ & $306 \pm 89$ & $310 \pm 112$ & $303 \pm 61$ & $152 \pm 52$ & $156 \pm 65$ & $148 \pm 35$ \\
\hline Walk (m) & $19466 \pm 2776$ & $18419 \pm 2842$ & $20512 \pm 2336^{\alpha}$ & $9691 \pm 1333$ & $9367 \pm 1409$ & $10015 \pm 1184^{\circ}$ \\
\hline $\operatorname{Jog}(\mathrm{m})$ & $19225 \pm 1341$ & $19087 \pm 1138$ & $19362 \pm 1536$ & $9575 \pm 846$ & $9617 \pm 802$ & $9534 \pm 899$ \\
\hline $\operatorname{Run}(\mathrm{m})$ & $10420 \pm 930$ & $10170 \pm 807$ & $10670 \pm 998$ & $5261 \pm 589$ & $5264 \pm 582$ & $5259 \pm 606$ \\
\hline High speed run (m) & $4689 \pm 691$ & $4583 \pm 466$ & $4795 \pm 861$ & $2393 \pm 412$ & $2335 \pm 327$ & $2452 \pm 480$ \\
\hline Maximum speed run (m) & $1107 \pm 308$ & $1147 \pm 288$ & $1067 \pm 329$ & $562 \pm 172$ & $561 \pm 160$ & $563 \pm 186$ \\
\hline Low intensity activity (m) & $38996 \pm 3197$ & $37817 \pm 3071$ & $40176 \pm 2939^{\alpha}$ & $19418 \pm 1551$ & $19140 \pm 1461$ & $19697 \pm 1609$ \\
\hline High intensity running (m) & $16216 \pm 1639$ & $15900 \pm 1243$ & $16532 \pm 1940$ & $8217 \pm 987$ & $8160 \pm 913$ & $8273 \pm 1067$ \\
\hline Very-high intensity running (m) & $5796 \pm 890$ & $5730 \pm 642$ & $5861 \pm 1098$ & $2955 \pm 521$ & $2896 \pm 423$ & $3015 \pm 604$ \\
\hline Team possession (n) & $37.3 \pm 4.6$ & $37.1 \pm 4.9$ & $37.6 \pm 4.3$ & $18.6 \pm 2.9$ & $18.4 \pm 3.4$ & $18.7 \pm 2.5$ \\
\hline Team possession $(\%)$ & $52.8 \pm 8.1$ & $56.6 \pm 6.2$ & $49.1 \pm 8.1^{\alpha}$ & $52.0 \pm 8.6$ & $55.1 \pm 8.0$ & $49.0 \pm 8.1^{\alpha}$ \\
\hline
\end{tabular}

Symbol $(\alpha)$ indicates significantly different $(p \leq 0.05)$ from winning using an independent samples t-test. Draws excluded from win/loss comparison during halves $(\mathrm{n}=2)$ and quarters $(\mathrm{n}=12)$.

\subsection{Half Analysis}

There were no significant differences between the first and second half in PlayerLoad ${ }^{\mathrm{TM}}$, collated distances, or in the number of team possessions, across all games or in relation to winning or losing (Table 2). There was a significant decrease in high 
speed running $(p=0.037)$ in the second half of full games lost (Figure 1).

Table 2. Team PlayerLoad ${ }^{\mathrm{TM}}$, collated distances and possession across halves, in relation to match outcome, mean $\pm S D$.

\begin{tabular}{|c|c|c|c|c|c|c|}
\hline \multirow{2}{*}{$\begin{array}{l}\text { Halves } \\
\text { Variable }\end{array}$} & \multicolumn{3}{|c|}{ All Games $(\mathbf{n}=\mathbf{2 0})$} & \multicolumn{3}{|l|}{ Winning (n=8) } \\
\hline & First Half & Second Half & Difference & First Half & Second Half & Difference \\
\hline PlayerLoad ${ }^{\mathrm{TM}}(\mathrm{AU})$ & $5330 \pm 296$ & $5215 \pm 322$ & $115 \pm 288$ & $5209 \pm 260$ & $5101 \pm 257$ & $108 \pm 317$ \\
\hline Total distance (m) & $55999 \pm 4442$ & $55195 \pm 3962$ & $803 \pm 2877$ & $53906 \pm 4496$ & $52666 \pm 3170$ & $1240 \pm 3743$ \\
\hline Low intensity activity (m) & $39279 \pm 3332$ & $38953 \pm 3113$ & $326 \pm 1996$ & $37993 \pm 3512$ & $37227 \pm 2788$ & $766 \pm 2189$ \\
\hline High intensity running (m) & $16550 \pm 1737$ & $16062 \pm 1558$ & $489 \pm 1501$ & $15912 \pm 1503$ & $15434 \pm 1245$ & $478 \pm 1890$ \\
\hline Very-high intensity running (m) & $5968 \pm 901$ & $5725 \pm 902$ & $243 \pm 643$ & $5765 \pm 652$ & $5617 \pm 730$ & $148 \pm 818$ \\
\hline Team possession (n) & $38.2 \pm 4.6$ & $36.1 \pm 4.4$ & $2.2 \pm 4.8$ & $40.6 \pm 3.2$ & $36.9 \pm 4.5$ & $3.8 \pm 4.9$ \\
\hline Team possession (\%) & $54.3 \pm 8.0$ & $51.2 \pm 7.7$ & $3.1 \pm 14.2$ & $54.8 \pm 6.4$ & $54.3 \pm 7.7$ & $0.6 \pm 12.5$ \\
\hline
\end{tabular}

Table 2. Continued.

\begin{tabular}{|c|c|c|c|}
\hline Halves & & Losing $(n=12)$ & \\
\hline Variable & First Half & Second Half & Difference \\
\hline PlayerLoad ${ }^{\mathrm{TM}}(\mathrm{AU})$ & $5410 \pm 301$ & $5291 \pm 349$ & $120 \pm 281$ \\
\hline Total distance (m) & $57394 \pm 3988$ & $56882 \pm 3599$ & $512 \pm 2269$ \\
\hline Low intensity activity (m) & $40135 \pm 3054$ & $40103 \pm 2861$ & $32 \pm 1898$ \\
\hline High intensity running (m) & $16976 \pm 1812$ & $16480 \pm 1652$ & $496 \pm 1272$ \\
\hline Very-high intensity running (m) & $6103 \pm 1039$ & $5797 \pm 1026$ & $306 \pm 527$ \\
\hline Team possession $(\mathrm{n})$ & $36.6 \pm 4.8$ & $35.5 \pm 4.3$ & $1.1 \pm 4.6$ \\
\hline Team possession (\%) & $53.9 \pm 9.1$ & $49.1 \pm 7.4$ & $4.8 \pm 15.5$ \\
\hline
\end{tabular}

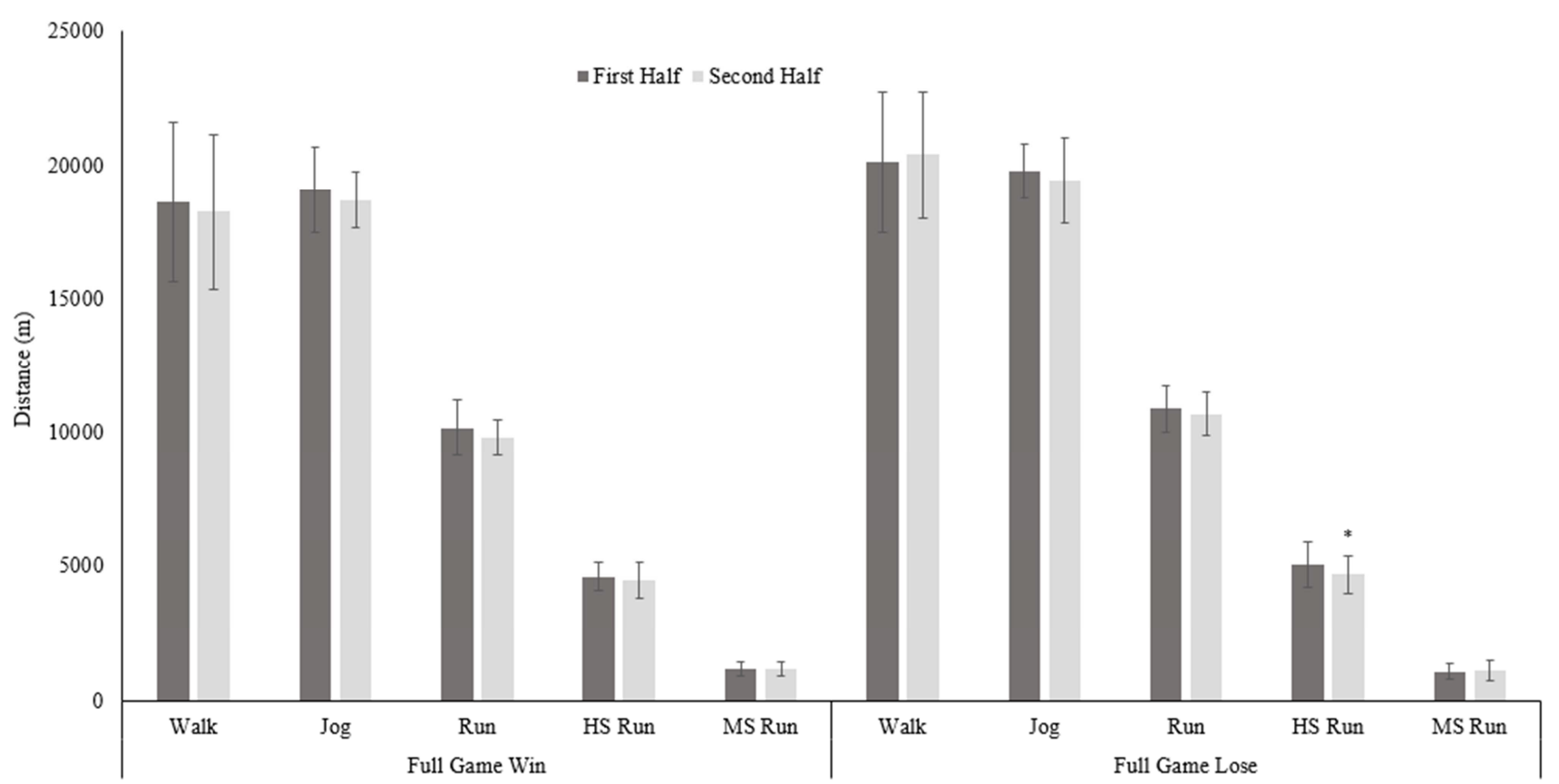

Figure 1. Differences in the activity profile across halves when match was won or lost, mean $\pm S D$. HS = high speed, MS = maximum speed. Symbol (*) indicates significantly different $(p \leq 0.05)$ from first half using a one sample $t$-test.

\subsection{Quarter Analysis}

In all games combined, there was a significant reduction in PlayerLoad ${ }^{\mathrm{TM}}(p=0.002)$, total distance $(p=0.006)$, jogging $(p=0.006)$, high speed running $(p=0.000)$, LIA $(p=0.025)$, HIR $(p=0.004)$, VHIR $(p=0.000)$, and in the frequency of team possession $(p=0.007)$ in Q4 compared to Q1 (Table 3). In winning games, there were no significant differences in any physical performance indices between Q1 and Q4, although a significant decline in the frequency of team possessions ( $p=0.032$ ) was observed (Table 4$)$. In contrast, no significant reduction in team possession was found in losing games. However, declines in PlayerLoad ${ }^{\mathrm{TM}}(p=$ $0.007)$, total distance $(p=0.022)$, HIR $(p=0.010)$ and VHIR $(p=0.001)$ were revealed in $\mathrm{Q} 4$, which coincided with significant reductions in jogging $(p=0.024)$ and high speed running $(p=0.000)$ (Figure 2). 
Table 3. Team physical performance and possession, across match quarters, mean $\pm S D$.

\begin{tabular}{|c|c|c|c|c|c|}
\hline \multicolumn{6}{|l|}{ All Games $(n=20)$} \\
\hline Variable & Quarter 1 (Q1) & Quarter 2 & Quarter 3 & Quarter 4 (Q4) & Difference Q1 - Q4 \\
\hline PlayerLoad ${ }^{\mathrm{TM}}(\mathrm{AU})$ & $2741 \pm 176$ & $2587 \pm 161$ & $2656 \pm 177$ & $2558 \pm 185^{*}$ & $184 \pm 224$ \\
\hline Total distance $(\mathrm{m})$ & $28603 \pm 2623$ & $27377 \pm 2144$ & $28177 \pm 2265$ & $27003 \pm 2085^{*}$ & $1600 \pm 2309$ \\
\hline Stand $(\mathrm{m})$ & $157 \pm 77$ & $143 \pm 36$ & $155 \pm 40$ & $148 \pm 28$ & $8 \pm 70$ \\
\hline Walk (m) & $9706 \pm 1398$ & $9799 \pm 1471$ & $9737 \pm 1418$ & $9781 \pm 1397$ & $-75 \pm 531$ \\
\hline $\operatorname{Jog}(\mathrm{m})$ & $10098 \pm 859$ & $9364 \pm 675$ & $9911 \pm 953$ & $9208 \pm 836^{*}$ & $890 \pm 1280$ \\
\hline $\operatorname{Run}(\mathrm{m})$ & $5413 \pm 718$ & $5165 \pm 510$ & $5249 \pm 478$ & $5085 \pm 541$ & $328 \pm 716$ \\
\hline High speed run (m) & $2551 \pm 404$ & $2316 \pm 402$ & $2441 \pm 380$ & $2159 \pm 349^{*}$ & $392 \pm 383$ \\
\hline Maximum speed run (m) & $591 \pm 156$ & $508 \pm 182$ & $589 \pm 171$ & $536 \pm 187$ & $55 \pm 171$ \\
\hline Low intensity activity (m) & $19961 \pm 1791$ & $19306 \pm 1719$ & $19803 \pm 1881$ & $19138 \pm 1506^{*}$ & $823 \pm 1508$ \\
\hline High intensity running (m) & $8555 \pm 1141$ & $7989 \pm 884$ & $8279 \pm 767$ & $7779 \pm 923^{*}$ & $775 \pm 1040$ \\
\hline Very-high intensity running $(\mathrm{m})$ & $3142 \pm 500$ & $2824 \pm 517$ & $3030 \pm 485$ & $2694 \pm 486^{*}$ & $447 \pm 464$ \\
\hline Team possession $(\mathrm{n})$ & $19.7 \pm 2.3$ & $18.6 \pm 3.2$ & $18.6 \pm 3.3$ & $17.5 \pm 2.7^{*}$ & $2.2 \pm 3.2$ \\
\hline Team possession (\%) & $53.1 \pm 9.5$ & $55.2 \pm 8.7$ & $52.1 \pm 7.8$ & $50.5 \pm 10.4$ & $2.6 \pm 17.1$ \\
\hline
\end{tabular}

Symbol $(*)$ indicates significantly different $(p \leq 0.05)$ from quarter 1 using a one sample t-test.

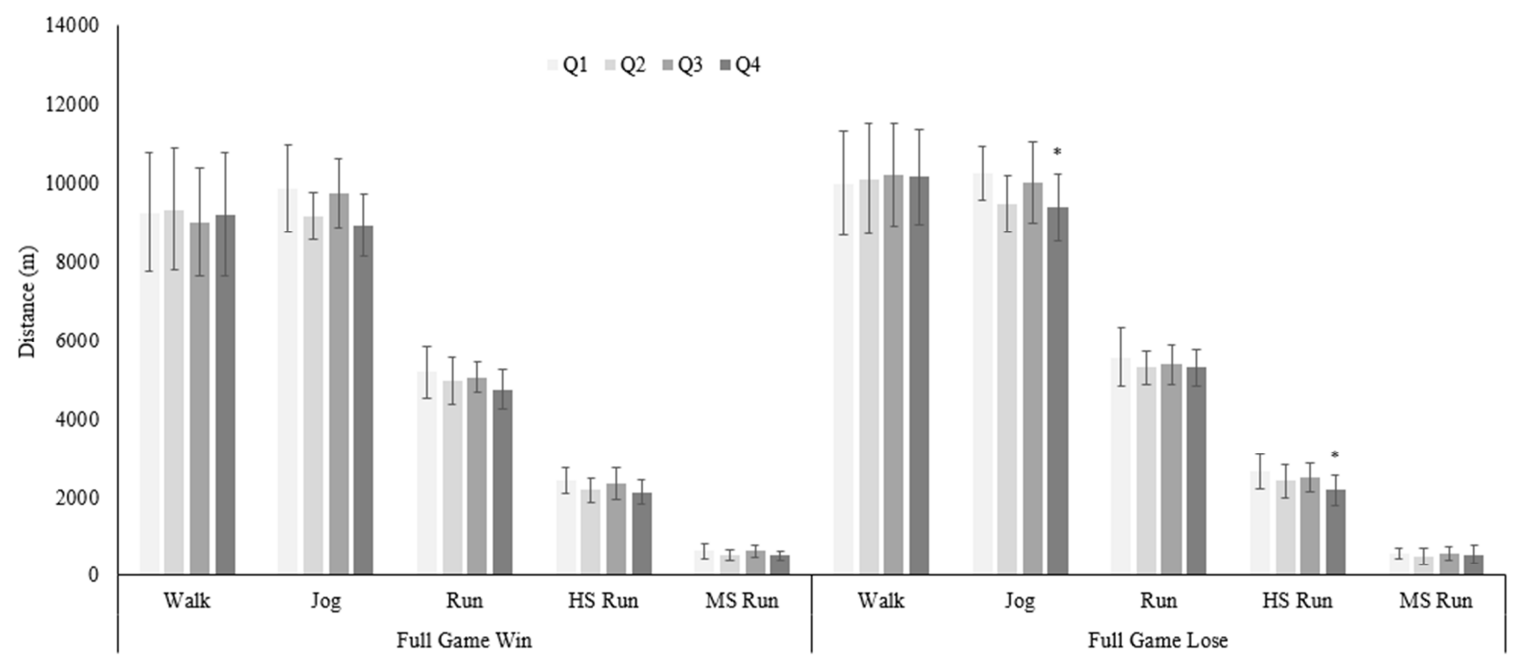

Figure 2. Differences in the activity profile across quarters ( $Q 1$ vs $Q 4)$ when match was won or lost, mean $\pm S D . Q 1=$ quarter $1, Q 2=$ quarter $2, Q 3=$ quarter 3, Q4 = quarter 4, HS = high speed run, MS = maximum speed run. Symbol (*) indicates significantly different ( $p \leq 0.05)$ from $Q 1$ using a one sample t-test.

Table 4. Team PlayerLoad ${ }^{\mathrm{TM}}$, collated distances and possession across quarters, in relation to match outcome, mean \pm SD.

\begin{tabular}{lllll}
\hline Quarters & Winning (n=8) & & & \\
\hline Variable & Quarter 1 & Quarter 2 & Quarter 3 & Quarter 4 \\
\hline PlayerLoadTM (AU) & $2675 \pm 157$ & $2534 \pm 123$ & $2604 \pm 114$ & Q1 - Q4 \\
Total distance (m) & $27565 \pm 2504$ & $26333 \pm 2121$ & $26948 \pm 1650$ & $25700 \pm 2234$ \\
Low intensity activity (m) & $19324 \pm 1746$ & $18664 \pm 1865$ & $18919 \pm 1502$ & $18295 \pm 1705$ \\
High intensity running (m) & $8241 \pm 953$ & $7670 \pm 833$ & $8030 \pm 695$ & $7400 \pm 803$ \\
Very-high intensity running (m) & $3058 \pm 445$ & $2707 \pm 390$ & $2969 \pm 457$ & $2647 \pm 363$ \\
Team possession (n) & $20.6 \pm 2.3$ & $20.0 \pm 2.0$ & $19.1 \pm 4.1$ & $17.8 \pm 2.2^{*}$ \\
Team possession (\%) & $54.5 \pm 7.3$ & $55.2 \pm 8.7$ & $54.5 \pm 8.9$ & $2.9 \pm 3.0$ \\
\hline
\end{tabular}

Table 4. Continue.

\begin{tabular}{|c|c|c|c|c|c|}
\hline Quarters & Losing $(n=12)$ & & & & \\
\hline Variable & Quarter 1 & Quarter 2 & Quarter 3 & Quarter 4 & Q1 - Q4 \\
\hline PlayerLoad ${ }^{\mathrm{TM}}(\mathrm{AU})$ & $2786 \pm 180$ & $2623 \pm 179$ & $2691 \pm 206$ & $2599 \pm 169^{*}$ & $187 \pm 197$ \\
\hline Total distance $(\mathrm{m})$ & $29295 \pm 2566$ & $28073 \pm 1939$ & $28996 \pm 2303$ & $27872 \pm 1508^{*}$ & $1423 \pm 1852$ \\
\hline Low intensity activity (m) & $20385 \pm 1763$ & $19734 \pm 1547$ & $20392 \pm 1930$ & $19700 \pm 1098$ & $686 \pm 1390$ \\
\hline High intensity running (m) & $8763 \pm 1246$ & $8202 \pm 886$ & $8445 \pm 796$ & $8032 \pm 942^{*}$ & $731 \pm 817$ \\
\hline Very-high intensity running $(\mathrm{m})$ & $3197 \pm 545$ & $2901 \pm 590$ & $3070 \pm 518$ & $2726 \pm 568^{*}$ & $472 \pm 348$ \\
\hline Team possession (n) & $19.0 \pm 2.0$ & $17.6 \pm 3.5$ & $18.2 \pm 2.9$ & $17.3 \pm 3.0$ & $1.7 \pm 3.3$ \\
\hline
\end{tabular}

$\mathrm{Q} 1=$ quarter $1, \mathrm{Q} 4=$ quarter 4. Symbol $(*)$ indicates significantly different $(\mathrm{p} \leq 0.05)$ from quarter 1 using a one sample t-test. 


\section{Discussion}

This is the first study to evaluate physical performance metrics and possession characteristics of an elite Gaelic football team in relation to winning or losing across discrete match periods. Through combining team performance data analysis, interpretations can be contextualised in a manner that is not possible when either physical or technical results are examined in isolation. Furthermore, the results extend recent analysis pertaining to specific aspects of both team [2, 16-18, 33] and player performances [15, 17, 34]. From a physical perspective, the main findings from this study reveal that total distance covered was higher in games and halves lost. Furthermore declines in PlayerLoad ${ }^{\mathrm{TM}}$, total distance, high speed running, HIR and VHIR were more pronounced when examined by match quarter compared to half and were only apparent in games lost. With regards technical performance, there was no difference in possession characteristics in relation to winning or losing games, however possession percentage declined in halves and quarters lost and possession frequency also declined in Q4 in all games combined. The implications of these findings in relation to both physical conditioning and technical practice are considered in the ensuing discussion. Performance variables that differentiated between winning and losing specific match periods and overall games are initially examined, prior to evaluating temporal changes that occurred across halves and quarters.

In interpreting the physical and technical performance profiles obtained in this study, it is important to first consider the tactical approaches employed by this team. To enhance the team's defensive organisation, some attacking players were often employed in roles characterising either an additional (third) midfielder or as defensive sweepers, whilst the remaining outfield players were regularly tasked with alternating between high and low defensive presses when not in possession. During offensive play, the team utilised a combination of long direct kicks, multiple short hand passes and/or carried the ball into the attacking zone, with the latter two strategies requiring players to perform repeated support runs, to pass or receive the ball. These tactical roles and strategies directly influenced the activity and technical profiles obtained from the players. Nevertheless, the fundamental aim of this team, which reflected most tactical strategies employed by coaches (excluding those premised on congested defences and damage limitation), was to gain or maintain possession, transfer the ball into the attacking zone to create scoring opportunities, and convert as many of these chances as possible into scores.

In the present study, the team covered significantly more total distance and performed more running in games lost. Similarly, in soccer it was reported that players from less successful teams competing at the bottom of the table, covered more total distance than teams ranked in the top five [35]. Furthermore, players covered a greater total distance when losing [36], and also covered a greater distance walking and jogging when playing against stronger teams [37]. In the current Gaelic football study, the total distance, walking and LIA, was higher in halves lost compared to halves won, supporting the findings previously highlighted from soccer $[36,37]$. In periods lost, it is unclear whether the increase in LIA (halves) and walking (halves and quarters) was due to fatigue, situational or psychological factors, or indeed a combination of these and/or other factors. In games lost, there was a small, but non-significant decline $(-3 \%)$ in the percentage of overall possession demonstrated by the team. However, there was a significant reduction in the percentage of possession in both halves $(-7.5 \%)$ and quarters $(-6.1 \%)$ lost, highlighting the importance of possession in contributing to determining the outcome of specific periods and potentially to the match. In soccer, possession was found to be greater when loosing than winning and was lower when playing against stronger opposition [37]. In Australian football, although there was no difference in time spent with or without possession reported between winning and losing full games, time spent in possession was higher than without possession in winning quarters [38]. Furthermore, the percentage time running at $>\sim 3.9$ and $>\sim 5.3 \mathrm{~m} \cdot \mathrm{s}^{-1}$ without possession was significantly greater in quarter wins than losses [38], indicating the contribution of this component to match period wins.

Although physical performance with- and withoutpossession was not evaluated in the present study, it is plausible that the significant increase in total distance and running in games lost, reflected a greater requirement of the players when not in possession to move into specific field positions (defensive formations) to deny space, thwart offensive manoeuvres and chase and pressurise the opposition in an effort to regain possession [39]. When losing, the players may have performed close to their maximal physical capacity in an attempt to draw or win the match [36]. In support of this, a recent study in Gaelic football demonstrated stronger positive correlations between the total distance ran by some playing positions and opposition time in possession, compared to the interaction of distance covered and time in possession reported by the reference team investigated [11]. Therefore, knowledge of the physical consequence of losing (or not having) possession, in terms of increased running requirements, can be used by coaches and fitness professionals to design scenarios and conditioning drills to emphasise the importance of both maintaining possession and also regaining possession as soon as possible when lost. Furthermore, having sufficient physical capacity to alternate the implementation of either a high or low defensive press can also be advantageous for players and may assist in this regard, as these tactical strategies were recently found to contribute to winning halves (low press) and quarters (high press) of games [18].

It was clear from evaluation of the temporal changes that occurred during the games that significant declines in team physical performance variables and possession were more pronounced in quarters compared to halves. The decrements in physical performance observed from Q1 to Q4 across all games replicate previous findings involving analysis of 
Gaelic football players, as declines in PlayerLoad ${ }^{\mathrm{TM}}$, HIR and heart rate responses in the last $15 \mathrm{~min}$ of games were recently reported [4], supporting similar findings of decrements in total distance covered and high speed running $\left(\geq 4.7 \mathrm{~m} \cdot \mathrm{s}^{-1}\right)$ distance in Q4 [9]. In Australian football, decrements from Q1 to Q4 were observed in $\operatorname{HIR}[5,19,21]$ and in total distance [19]. The declines in exercise intensity were suggested to be inevitable during the game and consequently higher intensity activities decreased in the latter stages, perhaps due to high levels of fatigue, although the influence of tactics and opponent performance was acknowledged as potential contributing factors [19]. Reductions in HIR $\left(>4.0-4.2 \mathrm{~m} \cdot \mathrm{s}^{-1}\right)$ through comparisons of the first and last 15 min periods [24] have also be reported in soccer, reinforcing the contention that fatigue occurs towards the end of games [40]. The postulated reduction in exercise intensity and observed decreases in LIA, HIR, and VHIR in the present study, perhaps explains the decline in PlayerLoad ${ }^{\mathrm{TM}}$, as this metric is influenced by; accelerations, locomotor activities and physical impacts. Additionally, the decrements in physical performance that occur towards the latter stages of games $[4,41]$ may coincide with a decrease in player density $[17,42]$ and manifest in a reduction in the intensity of man-to-man marking or incidence of physical contests. Interestingly, declines in physical performance in Q4 were observed in games that were lost but not in games that were won. It is unclear whether these decrements were due to fatigue or psychological factors, and/or contributed to the overall outcome. For example, a perception that the game was unwinnable, may have negatively impacted player motivation and subsequent work-rate [14] and resulted in a reduced effort to gain or regain possession [17]. The decrease in the frequency of team possessions overall in Q4 replicates the trend illustrated previously in both winning and losing Gaelic football teams [17], and may provide further evidence of a decline in match intensity [19]. Further, the higher baseline frequency of team possession in Q1 in winners compared to losers and the subsequent greater difference observed when compared to Q4, potentially explains why the decline was only significant in winners and not losers, although the increase in playing time reported in both the second half and Q4 in games lost may have contributed to this.

When the first half was compared to the second the only significant team physical or technical performance decline was a reduction in high speed running found in games lost. Similar reductions in high speed running were previously reported in intercounty hurling [23] and Australian soccer [25], whereas no difference was found in Gaelic football [4] or English soccer [24]. Various contextual factors may help to explain the discrepancies in studies, as some studies have failed to find a significant reduction in HIR during the second half [43], whereas others have reported declines in HIR [35, 44] and VHIR [35, 45]. Conflicting results were also reported in Australian football, with one study showing a decrease in HIR in the second half [19], although this trend was not replicated in a more recent investigation [5]. Unfortunately, none of these studies examined team performance or considered the data in relation to winning or losing. Nonetheless, the data obtained in the present study indicate that most physical performance variables did not decline significantly across halves, potentially influenced by the tactics, pacing and substitution strategy employed by the coaching team.

A limitation of this study is that performance data was evaluated from one team only. In addition, no between or within player analyses were conducted. Although a diverse range of physical performance metrics were included, only possession was analysed from a technical perspective. It is difficult to determine whether the decrease in the frequency or percentage of possession observed negatively impacted performance as the reference team may have become more efficient in their use of possession and conversion of possession into scoring opportunities and scores. To extend these findings, performance profiles incorporating a range of physical and technical variables, should be obtained from a large sample of teams (and individual players) competing across different competitions and analysed in relation to match score i.e., when winning, drawing or losing. Additional insights could be gained by evaluating contextual factors such as the influence of home advantage, level of opposition and stage of season on team performance and match outcome. Furthermore, weekly training loads could be evaluated to determine if the volume and intensity of prior training influenced match outcomes.

\section{Conclusion}

In this novel study alterations in team physical performance variables and possession were observed in match periods associated with either winning or losing. A greater total distance was covered in losing halves and full games. Furthermore, significant declines in physical performance were more pronounced when examined by match quarter compared to half and were also only apparent in games that were lost. Consequently, physical performance levels were generally maintained across halves and quarters in games that were won. Possession percentage declined in halves and quarters lost whereas possession frequency declined in Q4 in all games combined. Although not directly assessed, it is likely that match outcomes were influenced by levels of prior conditioning, previous training load, development of fatigue and/or various contextual/psychological factors. Examination of performance variables contributing to match and/or period outcome can therefore provide useful insights relating to aspects of performance that need to be addressed through a combination of physical, tactical and technical coaching and inform the between game training programmes and conditioning prescription. Further, the team performance benchmarks presented in this study, extends the current literature base and highlights the need for coaches to develop specific preparation strategies to address the decline in physical performance and possession observed particularly towards the latter stages of games (Q4). In addition to 
considering specific contextual factors, coaches and practitioners can use established team and player performance benchmarks to more effectively inform their ingame substitution strategies.

\section{Acknowledgements}

The authors would like to thank the players, coaches and members of the management team who facilitated the study.

\section{Disclosure Statement}

The authors declare that they have no competing interests.

\section{References}

[1] Beasley, K. J., 2015. Nutrition and Gaelic football: Review, recommendations, and future considerations. International Journal of Sport Nutrition and Exercise Metabolism, 25 (1): 1-13.

[2] Gamble, D., J. Bradley, A. McCarren and N. M. Moyna, 2019. Team performance indicators which differentiate between winning and losing in elite Gaelic football. International Journal of Performance Analysis in Sport, 19 (4): 478-490.

[3] Reilly, T. and D. Doran, 2001. Science and Gaelic football: A review. Journal of Sports Sciences, 19 (3): 181-193.

[4] Gamble, D., M. Spencer, A. McCarren and N. Moyna, 2019. Activity profile, PlayerLoad ${ }^{\mathrm{TM}}$ and heart rate response of Gaelic football players: A pilot study. Journal of Human Sport and Exercise, 14 (4): 1-15.

[5] Mooney, M., S. Cormack, B. O’Brien and A. J. Coutts, 2013. Do physical capacity and interchange rest periods influence match exercise-intensity profile in Australian football? International Journal of Sports Physiology and Performance, 8 (2): 165-172.

[6] Hughes, M. D. and R. M. Bartlett, 2002. The use of performance indicators in performance analysis. Journal of Sports Sciences, 20: 739-754.

[7] Cummins, C., R. Orr, H. O’Connor and C. West, 2013. Global positioning systems (GPS) and microtechnology sensors in team sports: A systematic review. Sports Medicine, 43 (10): $1025-1042$

[8] Malone, S., B. Solan, K. D. Collins and D. A. Doran, 2016. Positional Match Running Performance in Elite Gaelic Football. Journal of Strength and Conditioning Research, 30 (8): 2292-2298.

[9] Malone, S., B. Solan and K. Collins, 2017. The running performance profile of elite Gaelic football match-play. Journal of Strength and Conditioning Research, 31 (1): 30-36.

[10] Mangan, S., S. Malone, M. Ryan, J. McGahan, J. Warne, D. Martin, C. O'Neill, C. Burns and K. Collins, 2017. Influence of team rating on running performance in elite Gaelic football. Journal of Strength and Conditioning Research, 32 (9): 2584 2591.

[11] Mangan, S., M. Ryan, S. Devenney, A. Shovlin, J. McGahan, S. Malone, C. O'Neill, C. Burns and K. Collins, 2017. The relationship between technical performance indicators and running performance in elite Gaelic football. International Journal of Performance Analysis in Sport, 17 (5): 706-720.
[12] Mangan, S., S. Malone, M. Ryan, J. McGahan, C. O’Neill, C. Burns, J. Warne, D. Martin and K. Collins, 2017. The influence of match outcome on running performance in elite Gaelic football. Science and Medicine in Football, 1 (3): 272279 .

[13] Bradley, P. S. and T. D. Noakes, 2013. Match running performance fluctuations in elite soccer: Indicative of fatigue, pacing or situational influences? Journal of Sports Sciences, 31 (15): 1627-1638.

[14] Redwood-Brown, A. J., P. G. O'Donoghue, A. M. Nevill, C. Saward and C. Sunderland, 2019. Effects of playing position, pitch location, opposition ability and team ability on the technical performance of elite soccer players in different score line states. PLoS ONE, 14 (2): 1-21.

[15] Mangan, S., M. Ryan, A. Shovlin, J. McGahan, S. Malone, C. O'Neill, C. Burns and K. Collins, 2019. Seasonal changes in Gaelic football match-play running performance. Journal of Strength \& Conditioning Research, 33 (6): 1685-1691.

[16] Allister, A., P. J. Byrne, C. D. Nulty and S. Jordan, 2018. Game-related statistics which discriminate elite senior Gaelic football teams according to game outcome and final score difference. International Journal of Performance Analysis, 18 (4): 622-632.

[17] Gamble, D., A. McCarren, J. Bradley and N. Moyna, 2019. Temporal changes in team performance indicators which differentiate between winning and losing in elite Gaelic football. American Journal of Sports Science, 7 (4): 199-207.

[18] Gamble, D., A. McCarren, J. Bradley and N. Moyna, 2020. Novel component variables can be used to distinguish between winning and losing across match periods in elite Gaelic football. International Journal of Performance Analysis in Sport, ePub.

[19] Coutts, A. J., J. Quinn, J. Hocking, C. Castagna and E. Rampinini, 2010. Match running performance in elite Australian Rules Football. Journal of Science and Medicine in Sport, 13 (5): 543-548.

[20] Edwards, A. M. and T. D. Noakes, 2009. Dehydration: Cause of fatigue or sign of pacing in elite soccer? Sports Medicine, 39 (1): $1-13$.

[21] Duffield, R., A. J. Coutts and J. Quinn, 2009. Core temperature responses and match running performance during intermittent sprint exercise competition in warm conditions. Journal of Strength and Conditioning Research, 23 (4): 12381244 .

[22] Johnston, R. D., T. J. Gabbett, A. J. Seibold and D. G. Jenkins, 2014. Influence of physical contact on pacing strategies during game-based activities. International Journal of Sports Physiology and Performance, 9 (5): 811-816.

[23] Young, D., L. Mourot and G. Coratella, 2018. Match-play performance comparisons between elite and sub-elite hurling players. Sport Sciences for Health, 14 (1): 201-208.

[24] Bradley, P. S., W. Sheldon, B. Wooster, P. Olsen, P. Boanas and P. Krustrup, 2009. High-intensity running in English FA Premier League soccer matches. Journal of Sports Sciences, 27 (2): 159-168.

[25] Wehbe, G., T. Hartwig and C. Duncan, 2014. Movement analysis of Australian national league soccer players using global positioning system technology. Journal of Strength and Conditioning Research, 28: 834-42. 
[26] Boyd, Luke, J., K. Ball and R. J. Aughey, 2011. The reliability of MinimaxX accelerometers for measuring physical activity in Australian football. International Journal of Sport Physiology and Performance, 6: 311-321.

[27] Jennings, D., Cormack, S., Coutts, A. J., Boyd, L., \& Aughey, R. J., 2010. The validity and reliability of GPS units in team sport specific running patterns. International Journal of Sports Physiology and Performance, 5 (3): 328-341.

[28] Thornton, H. R., A. R. Nelson, J. A. Delaney, F. R. Serpiello and G. M. Duthie, 2019. Interunit reliability and effect of data-processing methods of global positioning systems. International Journal of Sports Physiology and Performance, 14 (4): 432-438.

[29] Luteberget, L. S., B. R. Holme and M. Spencer, 2018. Reliability of wearable inertial measurement units to measure physical activity in team handball. International Journal of Sports Physiology and Performance, 13 (4): 467-473.

[30] Beato, M., G. Coratella, A. Stiff and A. Dello Iacono, 2018. The validity and between-unit variability of GNSS units (STATSports apex 10 and $18 \mathrm{~Hz}$ ) for measuring distance and peak speed in team sports. Frontiers in Physiology, 9 (SEP):

[31] McGraw, K. O. and S. P. Wong, 1996. Forming inferences about some intraclass correlation coefficients. Psychological Methods, 1 (1): 30-46.

[32] Koo, T. K. and M. Y. Li, 2016. A guideline of selecting and reporting intraclass correlation coefficients for reliability research. Journal of Chiropractic Medicine, 15 (2): 155-163.

[33] McGahan, J., S. Mangan, K. Collins, C. Burns, T. Gabbett and C. O'Neill, 2018. Match-play running demands and technical performance among elite Gaelic footballers: Does divisional status count? Journal of Strength and Conditioning Research, Epub: doi: 10.1519/JSC.0000000000002450.

[34] McGahan, J., C. Burns, S. Lacey, T. Gabbett and C. O. Neill, 2018. An investigation in to the positional running demands of elite Gaelic football players. Journal of Strength and Conditioning Research, Epub: 10.1519/JSC.0000000000002492.

[35] Rampinini, E., F. M. Impellizzeri, C. Castagna, A. J. Coutts and U. Wisløff, 2009. Technical performance during soccer matches of the Italian Serie A league: Effect of fatigue and competitive level. Journal of Science and Medicine in Sport, 12 (1): $227-233$.
[36] Castellano, J., A. Blanco-Villaseñor and D. Álvarez, 2011. Contextual variables and time-motion analysis in soccer. International Journal of Sports Medicine, 32 (6): 415-421.

[37] Lago-Peñas, C., J. Lago-Ballesteros and E. Rey, 2011. Differences in performance indicators between winning and losing teams in the UEFA Champions League. Journal of Human Kinetics, 27 (1): 135-146.

[38] Gronow, D., B. Dawson, J. Heasman, B. Rogalski and P. Peeling, 2014. Team movement patterns with and without ball possession in Australian Football League players. International Journal of Performance Analysis in Sport, 14 (3): 635-651.

[39] Bradley, P. S., C. Lago-Peñas, E. Rey and A. Gomez Diaz, 2013. The effect of high and low percentage ball possession on physical and technical profiles in English FA Premier League soccer matches. Journal of Sports Sciences, 31 (12): $1261-1270$

[40] Waldron, M. and J. Highton, 2014. Fatigue and pacing in high-intensity intermittent team sport: An update. Sports Medicine, 44 (12): 1645-1658.

[41] Mohr, M., P. Krustrup and J. Bangsbo, 2003. Match performance of high-standard soccer players with special reference to development of fatigue. Journal of Sports Sciences, 21 (7): 519-528.

[42] Wallace, J. L. and K. I. Norton, 2014. Evolution of World Cup soccer final games 1966-2010: Game structure, speed and play patterns. Journal of Science and Medicine in Sport, 17 (2): 223-228.

[43] Bradley, P. S., M. Di Mascio, D. Peart, P. Olsen and B. Sheldon, 2010. High-Intensity Activity Profiles of Elite Soccer Players at Different Performance Levels. Journal of Strength \& Conditioning Research, 24 (9): 2343-2351.

[44] Carling, C. and G. Dupont, 2011. Are declines in physical performance associated with a reduction in skill-related performance during professional soccer match-play? Journal of Sports Sciences, 29 (1): 63-71.

[45] Di Salvo, V., W. Gregson, G. Atkinson, P. Tordoff and B. Drust, 2009. Analysis of high intensity activity in premier league soccer. International Journal of Sports Medicine, 30 (3): 205-212. 